\title{
SUPERCLUSTERS AND SUPERVOIDS
}

\author{
I. Kuneva and M. Kalinkov \\ Institute of Astronomy \\ Bulgarian Academy of Sciences \\ 72 Tsarigradsko Chausse blvd \\ 1784 Sofia \\ e-mail: markal@bgearn
}

\section{Introduction}

A new catalog of superclusters from the entire sky cluster catalog of Abell et al. (1989) is compiled. In fact the last version of Reference Catalog of ACO Clusters of Galaxies (Kalinkov and Kuneva 1994) is used. The RC contains information of optical, radio and X-ray observations gathered from catalogs, lists and papers.

There are more than 1000 ACO clusters with measured redshift. We have applied multiple regression analysis to obtain redshift estimate for the rest clusters. Estimators in the last version of the $\mathrm{RC}$ are almost the same as in Kalinkov et al. (1994).

We use measured as well as estimated redshift for luminosity distance to ACO clusters assuming $q_{0}=1 / 2$ and $H_{0}=100 \mathrm{~km} \mathrm{~s}^{-1} \mathrm{Mpc}^{-1}$.

\section{Catalog of Superclusters of Galaxies}

Our searching procedure for systems of galaxies is described by Kalinkov and Kuneva (1986) and Kuneva and Kalinkov (1990).

We use density contrast $f=10,20,40,100,200$, and 400. But we define $f=D_{\nu} / D_{100}$, where $D_{\nu}$ is the density in a supercluster with multiplicity $\nu$ and $D_{100}$ is the density in a sphere with $100 \mathrm{Mpc}$ radius around clusters. Evidently $D$ $\left[\mathrm{cl} \mathrm{Mpc}{ }^{-3}\right]$. Thus $f$ is a local density enhancement. After a supercluster is found $f$ refers to a mean local density enhancement over all supercluster members.

There are 893 superclusters with $\nu \geq 2$ in our catalog altogether. We list all superclusters for $|b| \geq 30^{\circ}$ and $R \leq 627 \mathrm{Mpc}(z=0.2)$ independently whether redshift is measured or estimated. A few superclusters with $|b|<30^{\circ}$ and $R>627$ $\mathrm{Mpc}$ are added - in all cases its cluster members have measured redshifts.

For $R \leq 627 \mathrm{Mpc}$ there are 302 superclusters with $b \geq 40^{\circ}$ and 394 with $b \leq-40^{\circ}$.

There are 4 superclusters with $R<60 \mathrm{Mpc}$ : Nr 173 (A262, 347, 426), Nr 246 (A3163, 3226), Nr 721 (A3656, 3698, 3706, 3742). Hydra-Centaurus supercluster is really remarkable - $\mathrm{Nr} 542(\mathrm{~A} 1060,3526,3537,3656,3574)$ - for $f=10-100$ the distance is $40 \mathrm{Mpc}$, while for $f=200-400$ is $33 \mathrm{Mpc}(\mathrm{A} 3526,3565)$ and for $f=200$ is $49 \mathrm{Mpc}(\mathrm{A} 3537,3574)$. 
The main Table contains data for superclusters with $\nu \geq 3$, having at least one cluster with measured redshift:

$\nu$ - multiplicity

$f$ - density contrast level

ACO - clusters

$R A(1950) D e c$ and $b$ - galactic latitude

$R$ - luminosity distance $\left(q_{0}=1 / 2, H_{0}=100 \mathrm{~km} \mathrm{~s}^{-1} \mathrm{Mpc}^{-1}\right), \mathrm{Mpc}$

$\triangle R A, \Delta D e c, \Delta R-$ corresponding extent, Mpc

$r_{\nu}$ - distance from the supercluster centre to the most distant member (radius; $r_{\nu}=\Delta r / 2$ for $\left.\nu=2\right), \mathrm{Mpc}$

$r_{\nu+1}$ - distance from the supercluster centre to the nearest cluster outside the configuration, Mpc

$D=D_{\nu} / D_{100}$ - ratio of the supercluster density to the mean local density, defined within a sphere of $100 \mathrm{Mpc}$ radius

$\mathcal{M}$ - supercluster mass in units $10^{14} \mathcal{M}_{\odot}$, according to the relation of Bahcall and Cen (1993)

\section{Catalog of Voids}

Operational deffinitions of minivoids (without any ACO-cluster in volume $V<10^{6}$ $\mathrm{Mpc}^{3}$ ) and of supervoids, together with searching procedures are given by Kuneva and Kalinkov (1991).

Catalogs of superclusters, minivoids and supervoids could be received from authors.

This work was supported in part by the National Scientific Research Funds (Contracts F107/1991 and F260/1992).

\section{References}

Abell, G. O., Corwin, H. G. and Olowin, R. P. 1989, $A p J$ 70, 1

Bahcall, N. A. and Cen, R. 1993, ApJ 407, L49

Kalinkov, M. and Kuneva, I. 1986, MNRAS 218, 49p

Kalinkov, M. and Kuneva, I. 1994, Reference Catalog of ACO Clusters of Galaxies (on magnetic tape), Institute of Astronomy, Bulg. Acad. Sci., Sofia

Kalinkov, M., Kuneva I. and Valtchanov I. 1994, ADASS III, eds. D. R. Crabtree, R. J. Hannisch G J. Barnes. ASP Conference Series (San Francisko), 61

Kuneva, I. and Kalinkov, M. 1990, Paired and Interacting Galaxies, eds. J. W. Sulentic, W. C. Keel \& C. M. Telesco (NASA), 143

Kuneva, I. and Kalinkov, M. 1991, Galaxy Environments and the Large Scale Structure of the Universe, eds. G. Giuricin, F. Mardirossian and M. Mezzetti (Trieste) 